\title{
Comparison of turbulence models for two- phase flow in a centrifugal separator
}

\author{
Erkin Madaliev ${ }^{1 *}$, Murodil Madaliev ${ }^{1}$, Kamol Adilov $^{2}$, and Tohir Pulatov ${ }^{3}$ \\ ${ }^{1}$ Fergana Polytechnic Institute, Fergana, Uzbekistan \\ ${ }^{2}$ Tashkent State Transport University, Tashkent, Uzbekistan \\ ${ }^{3}$ Turin Polytechnic University, Tashkent, Uzbekistan
}

\begin{abstract}
The numerical results of mathematical modeling of a twophase, axisymmetric swirling turbulent flow in the separation zone of a centrifugal separator are presented. The movement of the carrier gas flow was modeled using RANS, which were closed using the SARC turbulence models, the SST-RC model, and the SSG/LRR-RSM-w2012 model. For the numerical solution of the problem, the SIMPLE algorithm was used. The article compares the results of numerical calculation of turbulence models. The results of a comparison of numerical calculations with allowance for the effect of the solid phase on the dynamics of the air medium and without taking it into account with experimental data are presented.
\end{abstract}

\section{Introduction}

Centrifugal separators are widely used in many sectors of the national economy. The design of new models and the improvement of existing designs are closely related to analyzing the processes taking place inside these separators. The hydrodynamic flow pattern implemented in such devices has a significant impact on the entire technological process. Very often, in pneumatic separators, the air is used as a working medium, and the structures themselves have a complex geometric shape. In this case, the flow regimes are always turbulent and anisotropic. Physical modeling of such processes is expensive and time-consuming. Methods of mathematical modeling can be considered a promising way to successfully solve the problem of finding the velocity field while obtaining analytical solutions is almost impossible or associated with a low degree of reliability. Consequently, the only way to solve the problems posed is numerical simulation method [1,2].

There are basically three approaches for mathematical modeling of a two-phase turbulent flow: 1) direct modeling of turbulence based on the Navier-Stokes DNS equations $[3,4]$; 2) modeling of turbulence based on LES eddies [5]; 3) modeling of turbulence based on the Reynolds equations RANS. The modern capabilities of the DNS method with the determination of all components of the movement are limited by calculations at relatively low Reynolds numbers. In comparison with DNS, the LES method can be used to calculate flows with significantly higher Reynolds numbers. However, using the LES method for calculating near-wall flows requires either the introduction of additional "empiricism,"

*Corresponding author: madaliev.ME2019@mail.ru 
which consists of the parameterization of the layer adjacent to the wall, or the use of grids that approach the DNS method grids in their characteristics [6]. That is why the first and second approaches require a large computational resource. Therefore, we used turbulence models based on the RANS approach.

Thus, engineering calculations require turbulence models that fairly accurately describe the averaged fields and large-scale pulsations of swirling flows [7]. Many turbulence models, which have become widespread in engineering calculations, describe such flows poorly. To improve the adequacy of modeling turbulent swirling flows, attempts are made to modify the existing RANS turbulence models. In [8], Shur and Spalart proposed an amendment to the Spalart-Allmaras (SA) model named SARC. Subsequently, Smirnov and Menter in [9] generalized this correction to Menter's k- $\omega$ SST model (k- $\omega$ SST-RC model). The advantage of these models is that they describe very well a wide range of turbulence problems. The disadvantage of the Boussinesq hypothesis is that it is true for isotropic turbulence. However, anisotropic turbulence appears in a flow with strong rotation. In such cases, it is necessary to use the non-linear RANS approach. Therefore, the work also used a non-linear turbulence model - the Reynolds stress method SSG/LRR-RSM. The advantage of this model is that it well describes strongly swirling and separated flows.

In this work, a two-dimensional axisymmetric turbulent flow in an air centrifugal separator is considered, which is an important link in the processes of separation and classification of particles and obtaining powders of the required quality. The efficiency of the ongoing processes for separating powders into coarse and fine fractions will depend on how the flow structure is organized inside the working area. The aim of the undertaken numerical study is to clarify the nature of the swirl flow hydrodynamics for different geometries. A schematic diagram of a centrifugal separator is shown in Figure 1.

\subsection{Physical and mathematical statement of the problem}

The principle of operation of a centrifugal separator is presented in the articles $[10,15]$. It is easy to understand that the efficiency of this type of separator is highly dependent on its complex geometry. Therefore, to search for optimal geometric parameters, the problem arises of modeling the kinematics of particles inside the separator. It is clear that particle kinematics depends on the dynamics of the airflow. Therefore, two tasks arise here: 1) to study the dynamics of the airflow; 2) based on the obtained hydrodynamic parameters of the airflow, investigate the trajectories of the separated particles. In practice, the bulk density of dust in separators can be up to 18 . This value is significantly less than the density of incompressible air (1.29) [11]. Therefore, in many works, the influence of the solid phase on air dynamics is neglected. However, near the wall, where dust particles accumulate under the action of centrifugal force, the density of the solid phase can reach significant values. In this case, the effect of the solid phase on the dynamics of the gas phase cannot be neglected. Therefore, in this work, a numerical study of the turbulent flow is carried out, taking into account the effect of the solid phase on the dynamics of the airflow inside the centrifugal separator. For the numerical study of the problem posed, a system of equations is used, the Reynolds-averaged Navier-Stokes equations in a cylindrical coordinate system taking into account the interaction between phases [12]: 


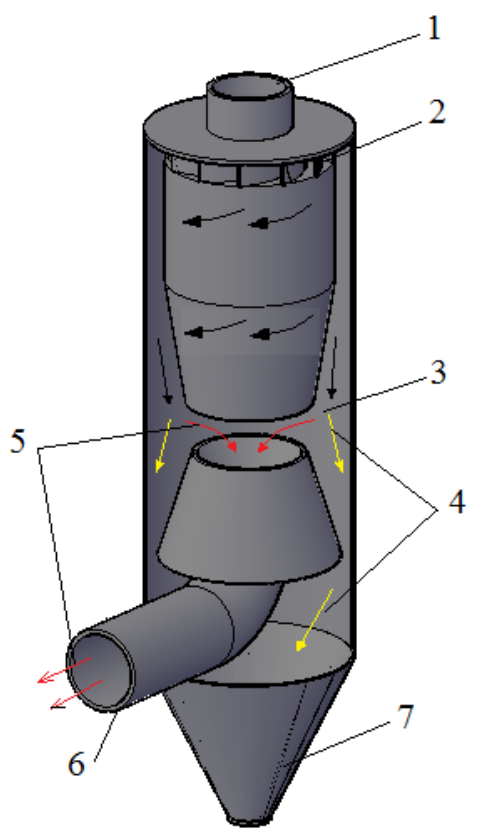

Fig.1. Scheme of the calculated air-centrifugal separator.

$$
\left\{\begin{array}{l}
\rho \frac{\partial \bar{U}_{i}}{\partial t}+\rho \bar{U}_{j} \frac{\partial \bar{U}_{i}}{\partial x_{j}}+\frac{\partial \bar{p}}{\partial x_{j}}=\mu \frac{\partial^{2} \bar{U}_{i}}{\partial x_{j} \partial x_{j}}+\frac{\partial}{\partial x_{j}}\left(-\overline{\rho v_{i}^{\prime} u_{j}^{\prime}}\right)-\sum_{k=1}^{N} \frac{\rho_{k}}{\rho} k_{k}\left(\bar{U}_{i}-\left(\bar{U}_{p k}\right)_{i}\right) \\
\frac{\partial\left(\bar{U}_{p k}\right)_{i}}{\partial t}+\bar{U}_{j} \frac{\partial\left(\bar{U}_{p k}\right)_{i}}{\partial x_{j}}=k_{k}\left(\bar{U}_{i}-\left(\bar{U}_{p k}\right)_{i}\right) \\
\frac{\partial \rho_{k}}{\partial t}+\bar{U}_{j} \frac{\partial \rho_{k}}{\partial x_{j}}=\frac{\partial}{\partial x_{j}}\left(\frac{\partial \rho_{k}}{\partial x_{j}}\right) \\
\frac{\partial \bar{U}_{i}}{\partial x_{j}}=0 .
\end{array}\right.
$$

here $\bar{U}_{i}$ is air flow velocity; $\left(\bar{U}_{p k}\right)_{i}$ is similar velocity components for the $k$-th dust fraction; $\bar{p}$ is hydrostatic pressure; $\rho$ is gas density; $\mu$ is its molecular viscosity; $\overline{v_{i}{ }^{\prime} u_{j}{ }^{\prime}}$ is components of the Reynolds stress tensor; $\rho_{k}$ is mass density of dust; $k_{k}$ is coefficient of interaction between air and the $k$-th fraction of dust; $N$ is the number of dust fractions.

The coefficient of interaction between phases is determined through the Stokes parameter for laminar flow: 


$$
k_{i}=\frac{18 \rho v}{\rho \rho \delta_{i}^{2}}
$$

Here $\rho_{\rho}$ is the density of the material of the dust particles, $\delta_{i}$ is the "effective" diameter of the particles. The initial and boundary conditions for the equation system (1) are set in a standard way [13]. The solution for the potential flow was used as an initial condition, for which the system of Euler equations was solved. Non-reflective boundary conditions were applied at the outer border. The adhesion condition was imposed on the surface of a solid. In the turbulence model SARC [14] and SST-RC, on the output, the Neumann condition was set; the Reynolds number depends on the input flow rate.

Since our computational domain is considered curvilinear and complex, to make the task easier, we change the coordinates [15] with the help of the transformation that is presented in [16].

Reynolds-averaged Navier-Stokes equations (2) are not closed. To close the system of equation (2), a linear approach was first used that uses the generalized Boussinesq hypothesis.

$$
-u_{i}^{\prime} u_{j}{ }^{\prime}=v_{t}\left(\frac{\partial U_{i}}{\partial x_{j}}+\frac{\partial U_{j}}{\partial x_{i}}\right)-\frac{2}{3} k \delta_{i j}
$$

Here $v_{t}$ is the turbulent viscosity. To find the turbulent viscosity, the SARC and SST$\mathrm{RC}$ turbulence models were used, taking into account the curvature of the streamlines.

Spalart-Allmaras models [14]. This model belongs to the class of one-parameter turbulence models. Here, only one additional equation appears for calculating the kinematic coefficient of eddy viscosity.

$$
\frac{\partial \tilde{v}}{\partial t}+U_{j} \frac{\partial \tilde{v}}{\partial x_{j}}=C_{b 1} f_{r 1} \tilde{S} \tilde{v}-C_{w 1} f_{w}\left(\frac{\tilde{v}}{d}\right)^{2}+\frac{1}{\sigma_{v}}\left(\frac{\partial}{\partial x_{j}}\left[(v+\tilde{v}) \frac{\partial \tilde{v}}{\partial x_{j}}\right]+C_{b 2}\left(\frac{\partial \tilde{v}}{\partial x_{j}} \frac{\partial \tilde{v}}{\partial x_{j}}\right)\right) .
$$

Turbulent eddy viscosity is calculated from:

In [9], a similar correction for the curvature of streamlines was applied to the $k$ - $\omega$ SST model (SST-RC model). The $f_{\text {rot }}$ correction is introduced into the generation members $k$ and $\omega$ :

$$
\left\{\begin{array}{l}
\frac{\partial k}{\partial t}+U_{j} \frac{\partial k}{\partial x_{j}}=\frac{\partial}{\partial x_{j}}\left[\left(v+\sigma_{k} v_{t}\right) \frac{\partial k}{\partial x_{j}}\right]+P f_{r 1}-\beta^{*} \omega k, \\
\frac{\partial \omega}{\partial t}+U_{j} \frac{\partial \omega}{\partial x_{j}}=\frac{\partial}{\partial x_{j}}\left[\left(v+\sigma_{\omega} v_{t}\right) \frac{\partial \omega}{\partial x_{j}}\right]+\frac{\gamma}{v_{t}} P f_{r 1}-\beta \omega^{2}+2\left(1-F_{1}\right) \frac{\sigma_{\omega 2}}{\omega} \frac{\partial \omega}{\partial x_{j}} \frac{\partial k}{\partial x_{j}}
\end{array}\right.
$$

where $P$ is the generation of energy of turbulent pulsations, the turbulent viscosity is calculated as 


$$
v_{t}=\frac{a_{1} k}{\left(a_{1} \omega, \Omega F_{2}\right)}
$$

The model is a Reynolds stress or non-linear model. An SSG/LRR model that uses an equation for the length scale equation $\omega$. Complete Reynolds stress model SSG / LRRomega (SSG / LRR-RSM-w2012) [17; 18] and one equation of the length scale are determined as follows:

$$
\begin{aligned}
& \frac{\partial R_{i j}}{\partial t}+\frac{\partial U_{k} R_{i j}}{\partial x_{k}}=P_{i j}+\Pi_{i j}-\varepsilon_{i j}+D_{i j}, \\
& \frac{\partial \omega}{\partial t}+\frac{\partial U_{k} \omega}{\partial x_{k}}=\frac{a_{\omega} \omega}{k} \frac{P_{k k}}{2}-B_{\omega} \omega^{2}+\frac{\partial}{\partial x_{k}}\left(\left(v+\sigma_{\omega} \frac{k}{\omega}\right) \frac{\partial \omega}{\partial x_{k}}\right)+\sigma_{d} \frac{1}{\omega} \frac{\partial k}{\partial x_{j}} \frac{\partial \omega}{\partial x_{j}} .
\end{aligned}
$$

here, $\rho R_{i j}=-\tau_{i j}=\rho u_{i}{ }^{\prime} u_{j}{ }^{\prime} P_{i j}$ is the generation of Reynolds stresses, $D_{i j}$ is the diffusion, $\varepsilon_{i j}$ is the dissipation, $\Pi_{i j}$ is the pressure redistribution term. The remaining values of the initial and boundary conditions are presented in [24, 25].

Modeling the kinematics of particle motion in a turbulent two-phase flow, there is no unified representation that would allow to correctly describe the object [11]. The model based on the concept of "trajectory dust particles" is considered incorrect due to the lack of consideration of interaction between Reynolds stresses and particles. On the other hand, there are undeniable advantages of the Lagrangian approach, which is closer to real processes and allows one to obtain the necessary information about the trajectories of particles, the residence time of particles in the apparatus, the minimum size of captured particles $[19,20,21,22]$. In this regard, in this work, the Lagrangian approach is used to model the efficiency of a centrifugal separator.

\section{Methods}

The numerical solution of the presented system of equations was carried out in physical variables velocity - pressure by a physical splitting of the velocity and pressure fields [23]. The numerical solution of the transport equation is carried out on a hybrid checkerboard difference grid by the control volume method. For the numerical solution of the transport equation, an upstream finite-difference scheme is used, which has a second-order accuracy. The Lagrange approach is convenient for calculating particle trajectories. For the numerical implementation of $U_{p}, V_{p}, W_{p}$ in equations (1), an implicit scheme was used. After the formation of the quasiperiodic regime, the nonstationary fields were averaged [26, 27].

\section{Results and Discussion}

In figure 3 illustrates the profiles of air velocities in different sections. Here are $U /$ Uref,$V /$ Uref, $W /$ Uref dimensionless speeds. Uref $=U_{0}$, Wref $=W_{0}$, Wref $/$ Uref $=0.85$. 

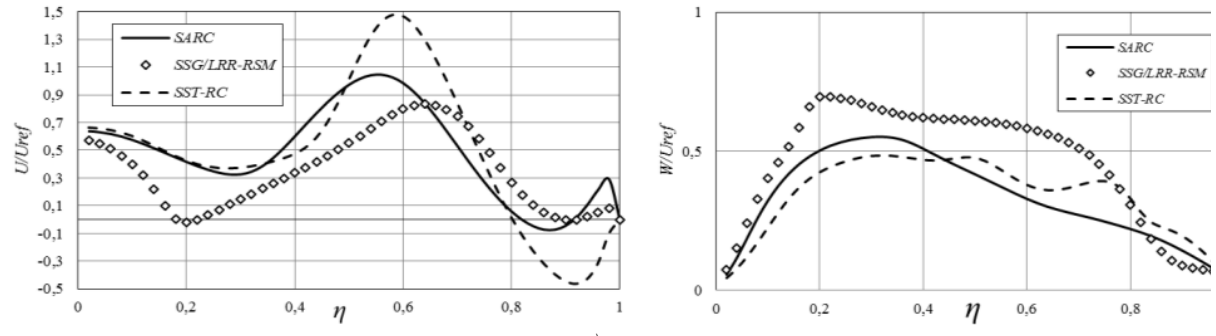

a)
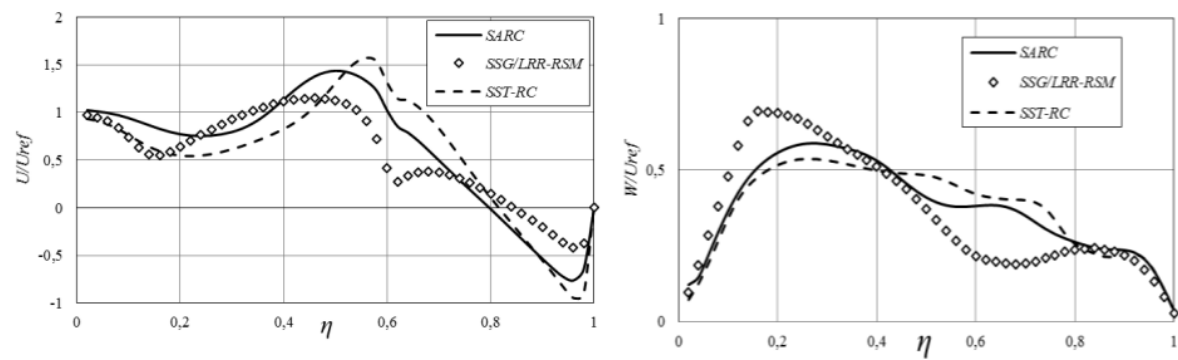

b)

Fig.3. Profiles, a) axial and tangential flow velocities in the section $\xi=0.65$, b) axial and tangential flow velocities in the section $\xi=0.7$.

In this task, the calculation accuracy was improved by using the SSG/LRR-RSM model. In fig. 4 shows a significant difference in the flow pattern obtained using different models. Calculation using the SSG/LRR-RSM method gives a longer length of recirculation zones, both on the axis and behind the step, compared to the calculation using the SARC and SST$\mathrm{RC}$ model.
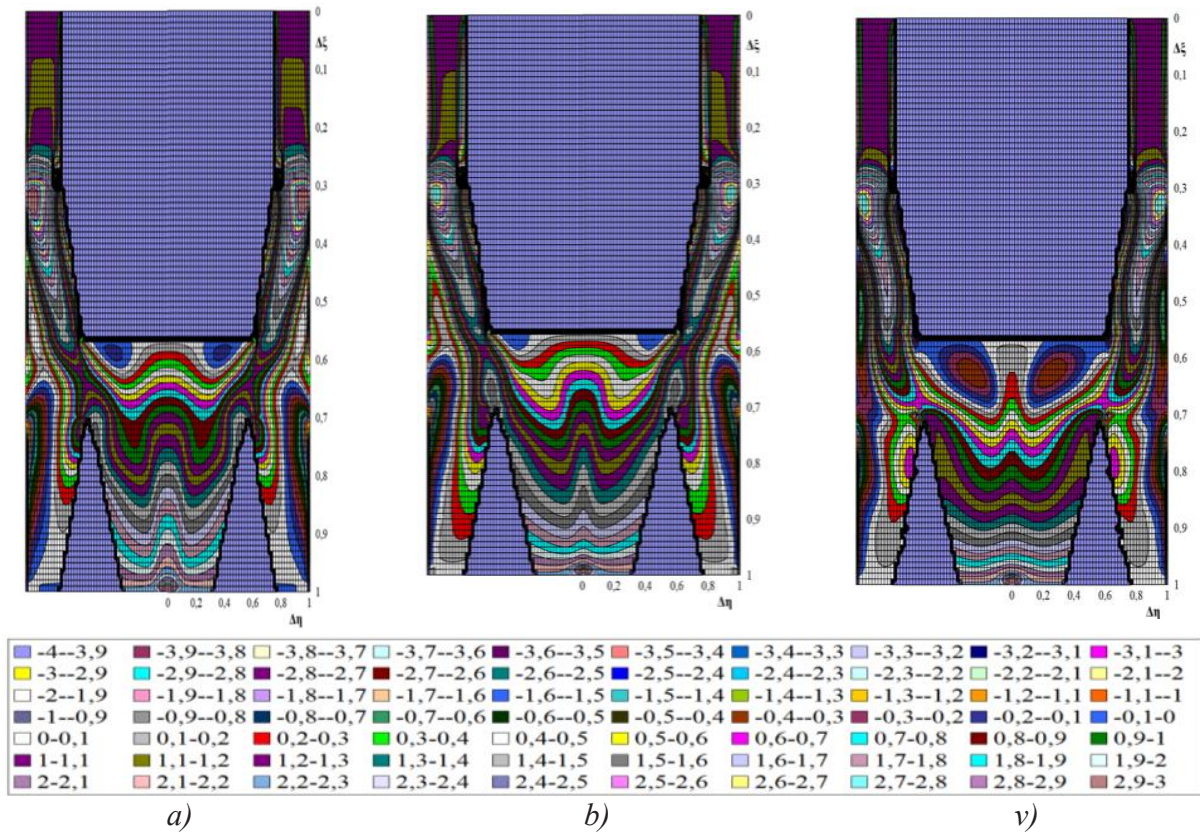

Fig.4. Isoline of longitudinal velocity along the separator length when using the turbulence model, a) SARC, b) SST-RC, c) SSG/LRR-RSM at $W / U_{\text {ref }}=0.85$ in dimensionless form. 
In figure 5 shows the dispersed composition of dust from the separator hopper according to the analyzer (rhombic points) for $U_{B x}=5.5 \mathrm{~m} / \mathrm{s}$ and according to the numerical calculation from the above-described non-linear SSG / LRR-RSM model (solid line), taking into account the effect of the solid phase on the air flow dynamics (dashed line), linear model SARC taking into account the influence of the solid phase on the airflow dynamics (crosses) and the SST-RC model taking into account the effect of the solid phase on the airflow dynamics at. $W_{\text {ref }} / U_{\text {ref }}=0.85$

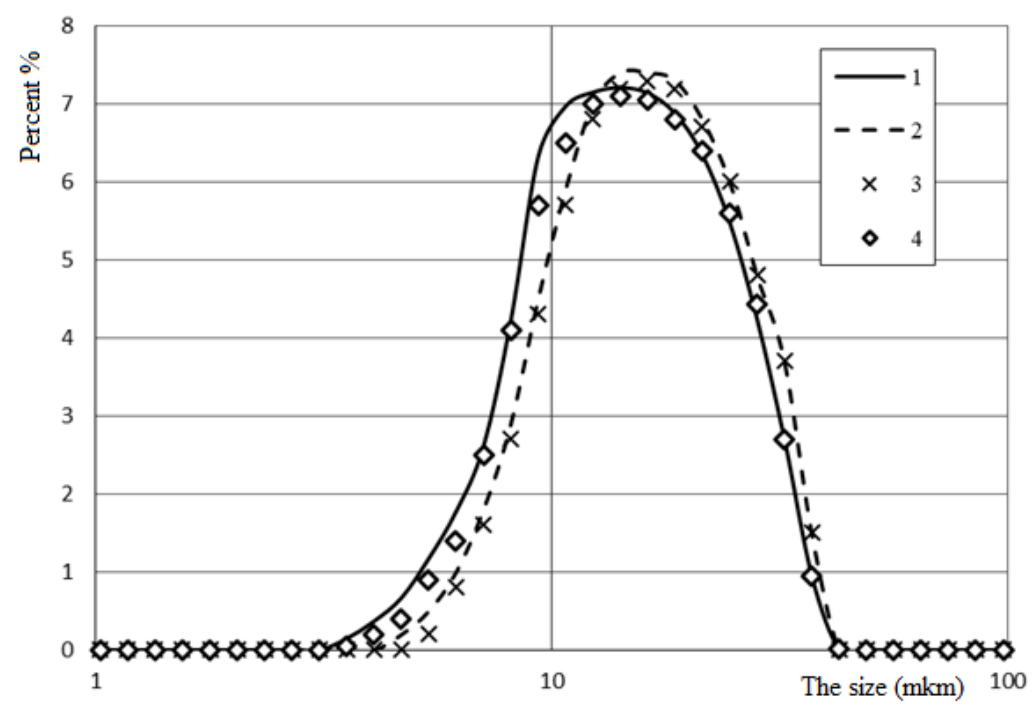

Fig.4. Disperse analysis of the composition of dust from the bunker of the separator. Taking into account the influence of the solid phase on the dynamics of the air flow according to the non-linear model SSG/LRR-RSM (1), according to the linear models SARC (2) and SST-RC (3), and according to experiment (4)

Dispersion analysis of the experiment was performed with a MALVERN laser analyzer. Comparisons show that the RSM non-linear turbulence model is more accurate than the linear turbulence models.

\section{Conclusions}

The considered problems of a centrifugal separator show that modeling of swirling turbulent flows requires a special approach to turbulence models. Since the swirling of the flow suppresses the generation of turbulence, the excessive turbulent viscosity at the inlet can lead to a significant distortion of the solution and the rapid erosion of the concentrated vortex. For the same reason, to obtain more correct results, turbulence models should be used to consider the swirl of the average flow. Nevertheless, linear models may not be enough for highly swirling flows to correctly predict the averaged velocity field. In this case, a non-linear model should be used. In particular, good results can be obtained using the Reynolds stress simulation method RSM. From (Fig. 4) it can be seen that the nonlinear RSM models describe swirling turbulent flows better than the linear SARC and SSTRC turbulence models. 


\section{References}

1. Schwab A.V, Brendakov V.N. Mathematical modeling of turbulent flow in a centrifugal apparatus, News of Tomsk Polytechnic University. 308, Russia, (2005).

2. Turubaev R.R, Schwab A.V. A numerical study of the aerodynamics of a swirling flow in a vortex chamber of a combined pneumatic apparatus, Tomsk State University Bulletin. (47). pp 87-98, Russia, (2017)

3. Versteegh T. A, Nieuwstadt T. M. Turbulent Budgets of Natural Convection in an Infinite, Differentially Heated, Vertical Channel, Intern. J. Heat Fluid Flow. 19. p. 135. (1997).

4. Boudjemadi R, Mairi V, LaurenceD, Le Quere P. Direct Numerical Simulation of Natural Convection in a Vertical Channel, A Tool for Second-Moment Closure Modelling, Proc. Engineering Turbulence Modelling and Experiments 3. Amsterdam: Elsevier. p. 39. (1996)

5. Peng S.-H., Davidson L. Large Eddy Simulation of Turbulent Buoyant Flow in a Confined Cavity, Intern. J. Heat Fluid Flow. 22. p. 323. (2001)

6. Cabot W., Moin P. Approximate Wall Boundary Conditions in the Large-Eddy Simulation of High Reynolds Number Flow, Flow, Turbulence and Combustion. (63). p 269. (1999)

7. Platonov D.V., Minakov A.V., Dekterev A.A., Numerical modeling of spatial flows with swirling flow, Computer Research and Modeling. 5. (4). pp. 635-648, Russia, (2013)

8. Spalart P.R., Shur M.L. On the sensitization of turbulence models to rotational and curvature, Aerospace Science and Technology. 1, (5). pp 297-302. (1997).

9. Smirnov P., Menter F. Sensitization of the SST turbulence model to rotation and curvature by applying the Spalart-Shur correction term, Proc. of ASME Turbo Expo 2008: Power for Land, Sea and Air, GT 2008, Germany, Berlin, June 9-13, p 10.(2008).

10. Malikov Z.M, Yuldashev A.T, Madaliev M.E. An experimental study of the effectiveness of a centrifugal air-passage separator, Problems of mechanics. pp 27-30, Uzbekistan, (2019)

11. Vasilevsky M.V., Zykov E.G. Calculation of the efficiency of gas purification in inertial apparatus, Tomsk: TPU Publishing House, p. 86, Russia

12. Loitsyansky L.G. The mechanics of fluid and gas, Moscow, Science. p. 840, Mexanika jidkocti i gaza, Moscow, Nauka, (1987)

13. Bradshaw P., Ferriss D.H., Atwell N.P. "Calculation of boundary layer development using the turbulent energy equation", J. Fluid Mech., 28 . pp 593-616. (1967).

14. Spalart P.R., Allmaras S.R. A one-equation turbulence model for aerodynamic flow, AIAA Paper. -12; (1). pp.439-478. (1992).

15. Madaliev M.E. Numerical simulation of the flow in a centrifugal separator based on the SA and SARC models, Mathematical modeling and numerical methods. № 2, p. 35-50, Russian, (2019)

16. Mises R., Zs. angew. Math. u. Mech., 7, p. 425. (1927).

17. Launder B.E., Reece G.J. and Rodi W. Progress in the development of a Reynolds stress turbulence closure. J. Fluid Mech. 68: pp 537-566. (1975).

18. Speziale C.G., Sarkar S., Gatski T.B. Modeling the pressure strain correlation of turbulence: an invariant dynamical systems approach. J. Fluid Mech. 227: pp 245272, (1991).

19. Shilyaev M.I., Shilyaev A.M. Modeling the process of dust collection in a oncethrough cyclone. 2 . The calculation of the fractional slip coefficient], Thermophysics and aeromechanics. 10. (3). pp 427-437, Russia, (2003) 
20. Shilyaev M.I., Shilyaev A.M. Modeling the process of dust collection in a oncethrough cyclone. 1. Aerodynamics and the diffusion coefficient of particles in a cyclone chamber, Thermophysics and Aeromechanics. 10. (2). pp 157-170, Russian, (2003)

21. Baranov D.A., Kutepov A.M., Lagutkin M.G. Calculation of separation processes in hydrocyclones], Theoretical Foundations of Chemical Technology. 30. (2). pp 117122, Russia, (1996)

22. Akhmetov T.G., Porfilyeva R.T., Gaysin L.G. Chemical technology of inorganic substances. - Prince 1. - M .: Higher school. p. 688, (2002).

23. Patankar S.V. Numerical Heat Transfer and Fluid Flow. Taylor and Francis. ISBN 978-0-89116-522-4, 1980.

24. Launder B.E., Spalding D.B. Lectures in Mathematical Models of Turbulence. London: Academic Press, p 169, (1972).

25. Julianne C.D, Jan-Renee C, "Evaluation of Full Reynolds Stress Turbulence Models in FUN3D", NASA/TM-2017-219468, pp 1-36, Texas, January 9-13, (2017).

26. M Usarov, G Mamatisaev, G Ayubov, D Usarov and D Khodzhaev. Dynamic calculation of boxed design of buildings. IOP Conf. Series: Materials Science and Engineering 883 (2020) 012186.https://doi.org/10.1088/1757-899X/883/1/012186, (2020)

27. M Usarov, G Ayubov, G Mamatisaev and B Normuminov. Building oscillations based on a plate model 2020 IOP Conf. Ser.: Mater. Sci. Eng. 883012211. https://doi.org/10.1088/1757-899X/883/1/012211, (2020). 